\title{
Serum bilirubin is associated with lung function in a Swiss general population sample
}

\author{
Ivan Curjuric ${ }^{1,2}$, Medea Imboden ${ }^{1,2}$, Martin Adam ${ }^{1,2}$, Robert W. Bettschart ${ }^{3}$, \\ Margaret W. Gerbase ${ }^{4}$, Nino Künzli1,2, Thierry Rochat ${ }^{4}$, Lucia Rohrer ${ }^{5}$, \\ Thomas B. Rothe ${ }^{6}$, Joel Schwartz 7 , Daiana Stolz ${ }^{8}$, Jean-Marie Tschopp?, \\ Arnold von Eckardstein ${ }^{5}$, Florian Kronenberg ${ }^{10}$ and Nicole M. Probst-Hensch ${ }^{1,2}$
}

Affiliations: 'Dept of Epidemiology and Public Health, Swiss Tropical and Public Health Institute, SwissTPH, Basel, ${ }^{2}$ University of Basel, Basel, ${ }^{3}$ Lungen Zentrum, Hirslanden Klinik, Aarau, ${ }^{4}$ Division of Pulmonary Medicine, University Hospitals, Geneva, ${ }^{5}$ Institute for Clinical Chemistry, University Hospital Zurich, Zurich, ${ }^{6}$ Dept of Internal Medicine and Pneumology, Zürcher Höhenklinik Davos, Davos Clavadel, ${ }^{8} \mathrm{Clinic}$ of Pulmonary Medicine and Respiratory Cell Research, University Hospital of Basel, Basel, and ${ }^{9}$ Centre Valaisan de Pneumologie, Centre Hospitalier du Centre du Valais, Crans-Montana, Switzerland. ${ }^{7}$ Harvard School of Public Health, Boston, MA, USA. ${ }^{10}$ Division of Genetic Epidemiology, Dept of Medical Genetics, Molecular and Clinical Pharmacology, Innsbruck Medical University, Innsbruck, Austria.

Correspondence: I. Curjuric, Dept of Epidemiology and Public Health, Chronic Disease Epidemiology Unit, Swiss Tropical and Public Health Institute SwissTPH, Socinstr. 57, 4051 Basel, Switzerland. E-mail: ivan.curjuricaunibas.ch

ABSTRACT Bilirubin is a strong antioxidant. Increased serum levels have been associated with lower respiratory disease and mortality risk. We studied the association of bilirubin with lung function in the Swiss study on Air Pollution and Lung Disease in adults (SAPALDIA) cohort.

Associations between natural logarithmised bilirubin and forced expiratory volume in $1 \mathrm{~s}$ (FEV1), forced vital capacity (FVC), FEV1/FVC and mean forced expiratory flow between 25\%-75\% of FVC (FEF25-75\%) were tested using multiple linear regression in the whole study population $(n=4195)$ and strata of eversmoking and high body mass index (BMI, defined by the highest distribution quartile). Associations were retested with single nucleotide polymorphism rs6742078, a genetic determinant of bilirubin.

High bilirubin levels were significantly associated with higher FEV1/FVC and FEF25-75\% overall. Upon stratification, significant associations persisted in ever-smokers, amounting to $1.1 \%$ (95\% CI $0.1-2.2 \%$ ) increase in FEV1/FVC, and $116.2 \mathrm{~mL} \cdot \mathrm{s}^{-1}\left(95 \% \mathrm{CI}-15.9-248.4 \mathrm{~mL} \cdot \mathrm{s}^{-1}\right)$ in $\mathrm{FEF} 25-75 \%$ per interquartile range of bilirubin exposure in smokers with high BMI. Associations were positive but nonsignificant in neversmokers with high BMI. Similarly, rs6742078 genotype TT was associated with increased FEV1/FVC and FEF25-75\%.

Our results suggest a possible protective role of bilirubin on lung tissue, which could be important for prevention and therapy.

@ERSpublications

Serum bilirubin is associated with lung function in subjects with low-grade inflammation from the general population http://ow.ly/tD3FP 


\section{Introduction}

Bilirubin is an end-product of heme degradation in the body that has raised considerable interest in research over the last decade [1]. Increased serum bilirubin has repeatedly been associated with decreased risks for coronary artery disease, myocardial infarction, peripheral arterial disease and stroke in retrospective and prospective clinical studies [2,3]. The evidence for beneficial effects of elevated serum bilirubin was supported by animal and in vitro experiments showing antioxidative and anti-inflammatory properties [4].

Beneficial effects of elevated serum bilirubin have also been observed on respiratory health. Higher bilirubin concentrations were associated with lower incidence of lung cancer, chronic obstructive lung disease and lung cancer mortality, as well as height-standardised lung function in previous population-based studies [5-7]. These results suggest that bilirubin might have protective effects in tissues exposed to the outer environment, such as the lungs, possibly by counteracting subclinical inflammation.

Furthermore, serum bilirubin levels were inversely associated with body mass index (BMI) [8], were found to be lower in smokers than never-smokers and decreased with higher smoking duration or intensity [9].

The major genetic determinant of serum bilirubin is UDP-glucuronosyltransferase 1A1 (UGT1A1) [10]. The enzyme degrades bilirubin by glucuronidation and allows its subsequent excretion into the bile. Polymorphisms in UGT1A1 explain $10-30 \%$ of serum bilirubin variation [1, 11]. A functional repeat polymorphism in the promoter region underlies Gilbert syndrome, which is prevalent in $5-10 \%$ of Caucasians. Compared with the wild-type allele consisting of six thymine-adenosine dinucleotides (TA) on each chromosome, persons homozygous for seven TA repeats have a 70\% decreased UGT1A1 activity [11]. A genome-wide study on bilirubin levels suggested that single nucleotide polymorphism (SNP) rs6742078 near UGT1A1 is in high linkage disequilibrium with the functional promoter repeat polymorphism, with its T-allele increasing bilirubin concentration [10].

Eight studies attempted to corroborate causality for the observed associations between bilirubin and cardiovascular outcomes using Mendelian randomisation, an instrumental variable analysis method, in which UGT1A1 variants are analysed to obtain unbiased estimates of bilirubin effects [12-19]. The most recent one on 67068 healthy controls and 11686 cases with ischaemic heart disease observed no significant association with genetically elevated bilirubin, even after meta-analysis with the earlier studies [19].

No comparable genetic studies have been conducted in the respiratory field, and the potential role of bilirubin in respiratory health has hardly been assessed. We therefore aimed to study whether bilirubin serum levels were associated with lung function in the general population sample of the Swiss study on Air Pollution and Lung Disease in adults (SAPALDIA) cohort study. The level of lung function is an early preclinical marker of underlying pathological lung processes such as inflammation and tissue remodelling. We a priori tested whether the relationship between bilirubin and lung function was altered by increased vulnerability arising from active tobacco smoking or subclinical inflammation related to excessive body weight. To assess causality for observed bilirubin associations, we studied the association of variant rs6742078 near UGT1A1 with lung function in a subset of the population.

\section{Methods \\ Design and study population}

SAPALDIA is a cohort study recruiting adults aged 18-60 years from eight Swiss communities. The SAPALDIA methods have been described in detail previously [20]. The present study is based on 4195 participants attending both examinations, with lung function testing, and with complete data on smoking exposure and bilirubin measurement (fig. 1).

Written informed consent was obtained from all study participants, and the study was approved by the Swiss Academy of Medical Sciences, as well as the respective regional ethics committees.

Received: March 282013 | Accepted after revision: Oct 062013 | First published online: Oct 312013

Support statement: The work was supported by the Swiss National Science Foundation (grant numbers 33CSCO-108796, 3247BO-104283，3247BO-104288，3247BO-104284，3247-065896，3100-059302，3200-052720，3200-042532，4026028099), the Federal Office for Forest, Environment and Landscape, the Federal Office of Public Health, the Federal Office of Roads and Transport, the canton's government of Aargau, Basel-Stadt, Basel-Land, Geneva, Luzern, Ticino, Valais and Zurich, the Swiss Lung League, the canton's Lung League of Basel Stadt/Basel Landschaft, Geneva, Ticino, Valais and Zurich, SUVA, Freiwillige Akademische Gesellschaft, UBS Wealth Foundation, Talecris Biotherapeutics GmbH, Abbott Diagnostics, European Commission 018996 (GABRIEL), Wellcome Trust WT 084703MA.

Conflict of interest: Disclosures can be found alongside the online version of this article at www.erj.ersjournals.com 


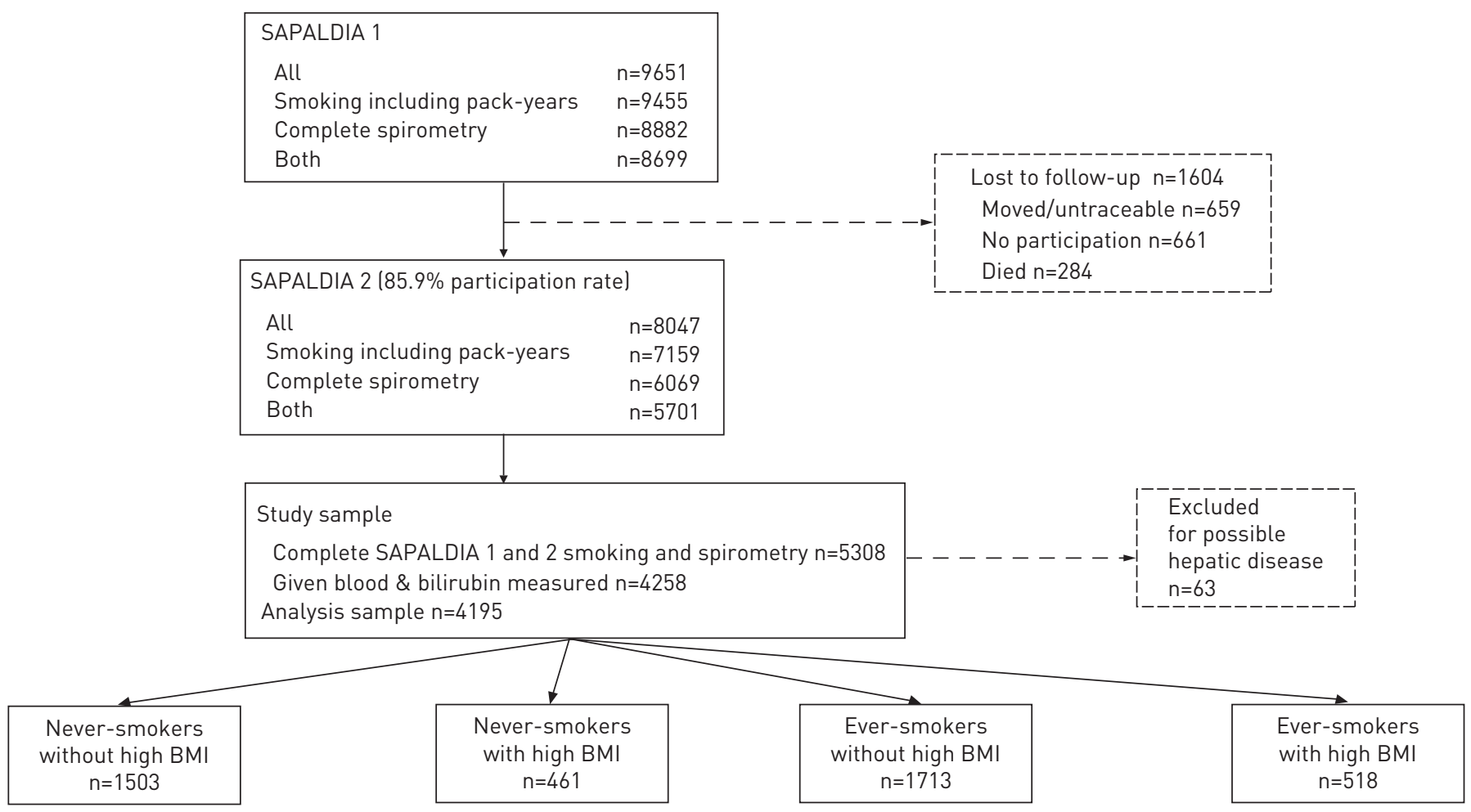

FIGURE 1 Selection of study participants. High body mass index (BMI) is defined as being in the highest quartile of the observed BMI distribution (BMI $\geqslant 28.36 \mathrm{~kg} \cdot \mathrm{m}^{-2}$. SAPALDIA: Swiss study on Air Pollution and Lung Disease in adults.

\section{Health questionnaire}

All study participants underwent an interview on smoking behaviour, exposure to second-hand smoke, occupational exposures, pre-existing respiratory, allergic and cardiovascular diseases and symptoms, as well as socioeconomic factors.

Education comprised highest attained school degree until follow-up examination and was grouped into three categories: low (primary school only), medium (high school) and high (university or college degree) educational level.

Never-smokers were defined as having smoked $<20$ packs of cigarettes or $360 \mathrm{~g}$ of tobacco during their lifetime. Former smokers had quit smoking at least 1 month before examination, and current smokers reported active smoking at the time of interview. In ever-smokers, pack-years of smoking were calculated by dividing the number of cigarettes per day by 20 , giving cigarette packs per day, which were multiplied by years of exposure.

Pre-existing cardiopulmonary disease was defined as self-declaration of heart disease, lung emphysema or chronic bronchitis, or use of health services for respiratory problems during the 12 months prior to followup examination. The detailed questions are given in the online supplementary material.

\section{$\mathrm{BMI}$ and spirometry}

Participants wore no shoes or heavy clothes for the measurement of weight and height. BMI was calculated by dividing weight (in kilograms) by the square of height (in metres).

Lung function testing was done using Sensormedics model 2200 (Sensormedics devices, Yorba Linda, CA, USA). Devices were calibrated daily, and comparability between devices was checked. Measurements were conducted according to the protocol of the European Community Respiratory Health Survey. Participants performed three to eight forced expiratory manoeuvres while sitting in an upright position. Manoeuvres had to comply with American Thoracic Society quality criteria [21], and at least two acceptable values for the forced expiratory volume in $1 \mathrm{~s}(\mathrm{FEV} 1)$ and forced vital capacity (FVC) were recorded. The ratio of FEV1/FVC was derived from measurements of the same manoeuvre. Mean forced expiratory flow at $25-75 \%$ of FVC (FEF25-75\%) was recorded from the curve displaying the highest sum of FEV1 and FVC. 


\section{Biomarkers}

Whole blood samples were collected in 2002, and serum blood markers were measured using a Modular P Autoanalyser (Roche Diagnostics, Rotkreuz, Switzerland). Total bilirubin was determined by the diazo method [22]. Liver enzyme activities were determined by enzymatic ultraviolet (UV) tests (including pyridoxalphosphate) for alanine aminotransferase (ALT) and aspartate aminotransferase (AST), and by photometric test for $\gamma$-glutamyl transferase (GGT). Coefficients of variation ranged from $1-3 \%$.

Bilirubin values $>17 \mu \mathrm{mol} \cdot \mathrm{L}^{-1}$ (upper normal value) with levels of ALT, AST or GGT equalling or surpassing the double of the upper normal value $\left(50,52\right.$ and $66 \mathrm{U} \cdot \mathrm{L}^{-1}$, respectively) were set to missing due to possible liver disease.

Genotype data for rs6742078 near UGT1A1 was acquired in the genome-wide GABRIEL asthma study for 982 nonasthmatic individuals $[23,24]$. Being nonasthmatic was defined as neither self-reported nor doctordiagnosed asthma using a standardised questionnaire [20]. Genotyping details are available in the online supplementary material.

\section{Definition of susceptible groups}

Two susceptibility conditions were considered regarding lung function. The first comprised ever-smoking. The second included high BMI because of the associated subclinical inflammation, which might interfere with the protective effects of bilirubin. For different reasons, this condition was defined statistically by using the highest quartile of the BMI distribution $\left(\mathrm{BMI} \geqslant 28.36 \mathrm{~kg} \cdot \mathrm{m}^{-2}\right.$ ) as threshold. First, waist circumference, more closely related to subclinical inflammation, was not available; and, secondly, categorising on the highest quartile was reasonable in absence of a validated BMI threshold to capture inflammation, as the common overweight definition of BMI $\geqslant 25 \mathrm{~kg} \cdot \mathrm{m}^{-2}$ was met by a large proportion of our older-aged study population (reflected by a mean BMI of $25.7 \mathrm{~kg} \cdot \mathrm{m}^{-2}$ ). Obesity $\left(\mathrm{BMI} \geqslant 30 \mathrm{~kg} \cdot \mathrm{m}^{-2}\right.$ ) was not chosen because of low sample size and its mechanical effects on lung function. Categorising BMI also enabled subgroup definitions in combination with ever-smoking.

We defined three susceptibility groups: never-smokers with high BMI, ever-smokers without, and eversmokers with high BMI. Never-smokers without high BMI served as the reference group.

\section{Statistical analysis}

The distribution of the following was tabulated for the whole study population and susceptible groups: sex, age, educational level, smoking status, pack-years smoked, height, weight, BMI, FEV1, FVC, FEV1/FVC, FEF25-75\%, pre-existing cardiopulmonary disease, bilirubin, C-reactive protein levels and genotypes of UGT1A1 SNP rs6742078. To account for differential distribution of sex and age, adjusted bilirubin mean concentrations and standard errors were calculated for each group by predicting the values from a multiple linear regression model of log bilirubin on sex, age and group membership. Predictions were exponentiated to give units of $\mu \mathrm{mol} \cdot \mathrm{L}^{-1}$.

Bilirubin values were transformed using the natural logarithm to achieve a more symmetric distribution. The cross-sectional association between log-transformed bilirubin values and FEV1, FVC, FEV1/FVC and FEF25-75\% from year 2002 was assessed using multiple linear regression adjusting stepwise for sex, age, height and weight, then educational level and study area, and finally ever-smoking status and pack-years. To check for interactions, dummy variables coding susceptibility group membership and multiplicative terms with $\log$ bilirubin were included into the models. Analyses were then stratified by susceptibility groups. Never-smoking participants without high BMI served as the reference group.

As an analysis of sensitivity, participants with elevated bilirubin levels (but normal liver enzymes) were excluded to assess whether the observed associations persisted in the normal range of serum bilirubin. Analyses were also repeated after excluding participants taking asthma medication (defined as a positive answer to the question "Are you currently taking any medicines including inhalers, aerosols or tablets for asthma?"). Finally, analysis was stratified by the presence of cardiopulmonary disease to assess the role of pre-existing pathologies on the associations under study. Interaction was tested analogously with multiplicative interaction terms.

Mendelian randomisation analysis is based on the idea that during meiosis, the alleles of a genetic locus are passed on to the next generation by chance. If the genetic locus highly influences the levels of a biomarker, this natural randomised experiment allows inferences to be made on the causality of biomarker effects [25]. Accordingly, the association between rs6742078 genotypes and serum bilirubin was tested using Wilcoxon rank sum tests. Associations between rs6742078 genotypes and lung function were tested in regression models with the same covariates as for serum bilirubin analysis. Recessive models were analysed (allele TT 
against GG/GT) to correspond with the functional impact of UGT1A1 promoter (TA) 7 homozygosity [3]. In view of the small sample size $(\mathrm{n}=982)$ no stratified analysis was run.

All analyses were conducted using STATA version 10.1 (StataCorp, College Station, TX, USA). Adjusted mean bilirubin concentrations and standard errors were predicted using the "adjust" post-regression estimation command. Two-sided $\alpha$-values of 0.05 and 0.1 were chosen as significance thresholds for main effects and interactions, respectively.

\section{Results}

Characteristics of the study population

Our study comprised 4195 subjects, of whom 1503 never-smokers without and 461 with high BMI, and 1713 ever-smokers without and 518 with high BMI. Overall, 53.2\% were females, $22.1 \%$ were current smokers and $31.0 \%$ were former smokers (table 1). Average age was 51.9 years, and the mean FEV1, FVC and $\mathrm{FEF} 25-75 \%$ were $3.2 \mathrm{~L}, 4.2 \mathrm{~L}$, and $2.6 \mathrm{~L} \cdot \mathrm{s}^{-1}$, respectively. Median tobacco smoke exposure in eversmokers was 17.0 pack-years (interquartile range 6-35). Median serum bilirubin in all participants was $7 \mu \mathrm{mol} \cdot \mathrm{L}^{-1}$ (interquartile range $5-10 \mu \mathrm{mol} \cdot \mathrm{L}^{-1}$ ). Across susceptibility groups, FEV1/FVC and FEF $25-75 \%$ decreased with high BMI and smoking exposure. Groups with high BMI were older and less educated, while more males were in the smoking groups. Bilirubin serum values were apparently comparable across the groups, but after adjusting for differences in sex and age distribution, concentrations decreased progressively from 7.84 to $6.77 \mu \mathrm{mol} \cdot \mathrm{L}^{-1}$ with increasing BMI and smoking exposure (online supplementary table S1).

\section{Associations of bilirubin with lung function values}

In the basic analysis models adjusting for sex, age, height and weight, FEV1, FEV1/FVC and FEF25-75\% were significantly and positively associated with serum bilirubin (table 2). One log unit increase in bilirubin, which corresponds to a 2.72 -fold increase in serum concentration, was associated with increases as follows ( $\beta$ estimate (95\% confidence interval)): FEV1 36.4 (6.7-66.1) mL; FEV1/FVC 0.9 (0.5-1.4)\%; and FEF25-75\% $115.2(58.5-172.0) \mathrm{mL} \cdot \mathrm{s}^{-1}$. Effect estimates decreased after adjustment for tobacco smoke exposure, which also resulted in a loss of statistical significance for the FEV1 association. In contrast, FEV1/FVC and FEF25-75\% associations remained significant with estimated increases of $0.5(0.1-1.0) \%$ and $66.9(11.4-122.5) \mathrm{mL} \cdot \mathrm{s}^{-1}$ per log unit bilirubin. This corresponds to increases of $0.3(0.1-0.7) \%$ in FEV1/FVC and 46.4 (7.9-84.9) $\mathrm{mL} \cdot \mathrm{s}^{-1}$ in $\mathrm{FEF} 25-75 \%$ over the observed interquartile exposure range $\left(5-10 \mu \mathrm{mol} \cdot \mathrm{L}^{-1}\right.$ bilirubin, i.e. a doubling concentration).

For FEV1/FVC, the interaction between log bilirubin and being an ever-smoker with or ever-smoker without high BMI was statistically significant ( $p_{\text {interaction }} 0.007-0.023$, data not shown). Interactions were also

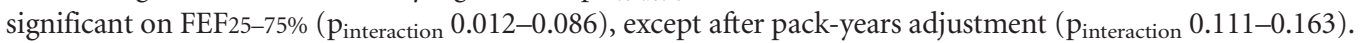
Stratifying the analysis by susceptibility groups confirmed the interactions (table 3): no significant associations were present in never-smokers without high BMI. In never-smokers with high BMI, higher, although nonsignificant $\beta$ estimates were observed for FEV1, FEV1/FVC and FEF25-75\%. Associations between $\log$ bilirubin and FEV1, FEV1/FVC and $\mathrm{FEF} 25-75 \%$ were significant in ever-smokers without high BMI. They did not withstand pack-years adjustment for FEV1 and FEV1/FVC (21.7 (-23.6-67.0) mL and $0.6(-0.1-1.2) \%$ increase per log unit bilirubin, respectively), but only FEF25-75\% (88.7 (3.6-173.8) $\left.\mathrm{mL} \cdot \mathrm{s}^{-1}\right)$. The largest $\beta$ estimates were observed in ever-smokers with high BMI across all FEV1/FVC and FEF25-75\% models. After pack-years adjustment, each log unit bilirubin was significantly associated with $1.6(0.1-3.2) \%$ higher FEV1/FVC and marginally associated with $167.6(-23.0-358.3) \mathrm{mL} \cdot \mathrm{s}^{-1}$ higher FEF25-75\%. Over the observed interquartile range of bilirubin, this corresponds to increases of $1.1(0.1-2.2) \% \mathrm{FEV} 1 / \mathrm{FVC}$ and $116.2(-15.9-248.4) \mathrm{mL} \cdot \mathrm{s}^{-1} \mathrm{FEF} 25-75 \%$.

\section{Sensitivity analyses}

Association patterns were not substantially affected by the exclusion of participants with bilirubin levels above the normal range or those with asthma medication affected (fig. 2). Significant associations were observed in persons with and without pre-existing cardiopulmonary disease (online supplementary table S2). For FEV1/FVC, estimates were significantly higher in the diseased group for education- and smoking-adjusted models ( $p_{\text {interaction }} 0.086$ and 0069, respectively; data not shown).

\section{Associations of rs6742078 T-alleles with serum bilirubin and lung function values}

Serum bilirubin values differed significantly according to the number of rs6742078 T-alleles (online supplementary table S3). Median concentrations were 6 and $7 \mu \mathrm{mol} \cdot \mathrm{L}^{-1}$ for gentoypes GG and GT, versus $13 \mu \mathrm{mol} \cdot \mathrm{L}^{-1}$ for homozygous T-alleles (p-value for Wilcoxon rank sum test $<0.001$ ).

Homozygous carriers of the rs6742078 T-allele had significantly higher levels of FEV1/FVC and FEF25-75\% in progressively adjusted linear regression models (table 4), resulting in 1.7 (0.4-3.0)\% higher FEV1/FVC 
TABLE 1 Characteristics of the whole study sample and analysis subgroups

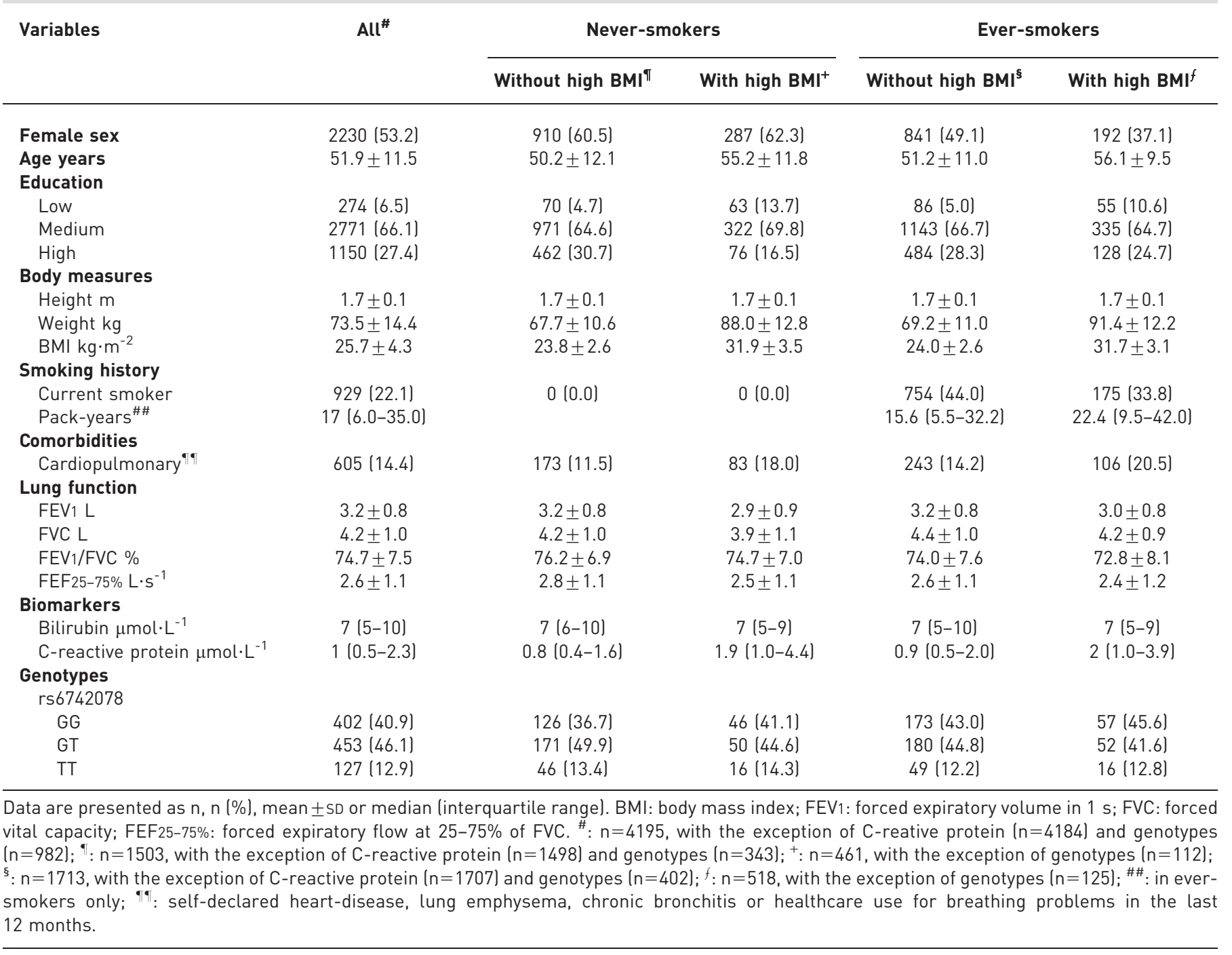

TABLE 2 Association of logarithmised bilirubin with lung function parameters in the whole study population

\begin{tabular}{|c|c|c|c|}
\hline Outcome & Model covariates & $\beta$ estimate $(95 \% \mathrm{Cl})$ & p-value \\
\hline \multirow[t]{3}{*}{ FEV1 $\mathrm{mL}$} & Sex, age, height, weight & $36.4(6.7-66.1)$ & 0.016 \\
\hline & All previous and education, study area & $38.0(8.4-67.6)$ & 0.012 \\
\hline & All previous and ever-smoking, total pack-years & $13.8(-15.5-43.2)$ & 0.356 \\
\hline \multirow[t]{3}{*}{ FVC mL } & Sex, age, height, weight & $-6.4(-41.4-28.7)$ & 0.722 \\
\hline & All previous and education, study area & $-4.9(-39.6-29.7)$ & 0.781 \\
\hline & All previous and ever-smoking, total pack-years & $-14.5(-49.3-20.4)$ & 0.416 \\
\hline \multirow[t]{3}{*}{ FEV1/FVC \% } & Sex, age, height, weight & $0.9(0.5-1.4)$ & $<0.001$ \\
\hline & All previous and education, study area & $1.0(0.5-1.4)$ & $<0.001$ \\
\hline & All previous and ever-smoking, total pack-years & $0.5(0.1-1.0)$ & 0.012 \\
\hline \multirow[t]{3}{*}{ FEF $25-75 \% \mathrm{~mL} \cdot \mathrm{s}^{-1}$} & Sex, age, height, weight & $115.2(58.5-172.0)$ & $<0.001$ \\
\hline & All previous and education, study area & $118.0(61.9-174.1)$ & $<0.001$ \\
\hline & All previous and ever-smoking, total pack-years & $66.9(11.4-122.5)$ & 0.018 \\
\hline
\end{tabular}

Estimates are per natural log unit increase in bilirubin. Positive values mean higher lung function and negative values mean lower function. $\mathrm{FEV} 1$ : forced expiratory volume in 1 s; FVC: forced vital capacity; FEF25-75\%: forced expiratory flow at 25-75\% of FVC. 


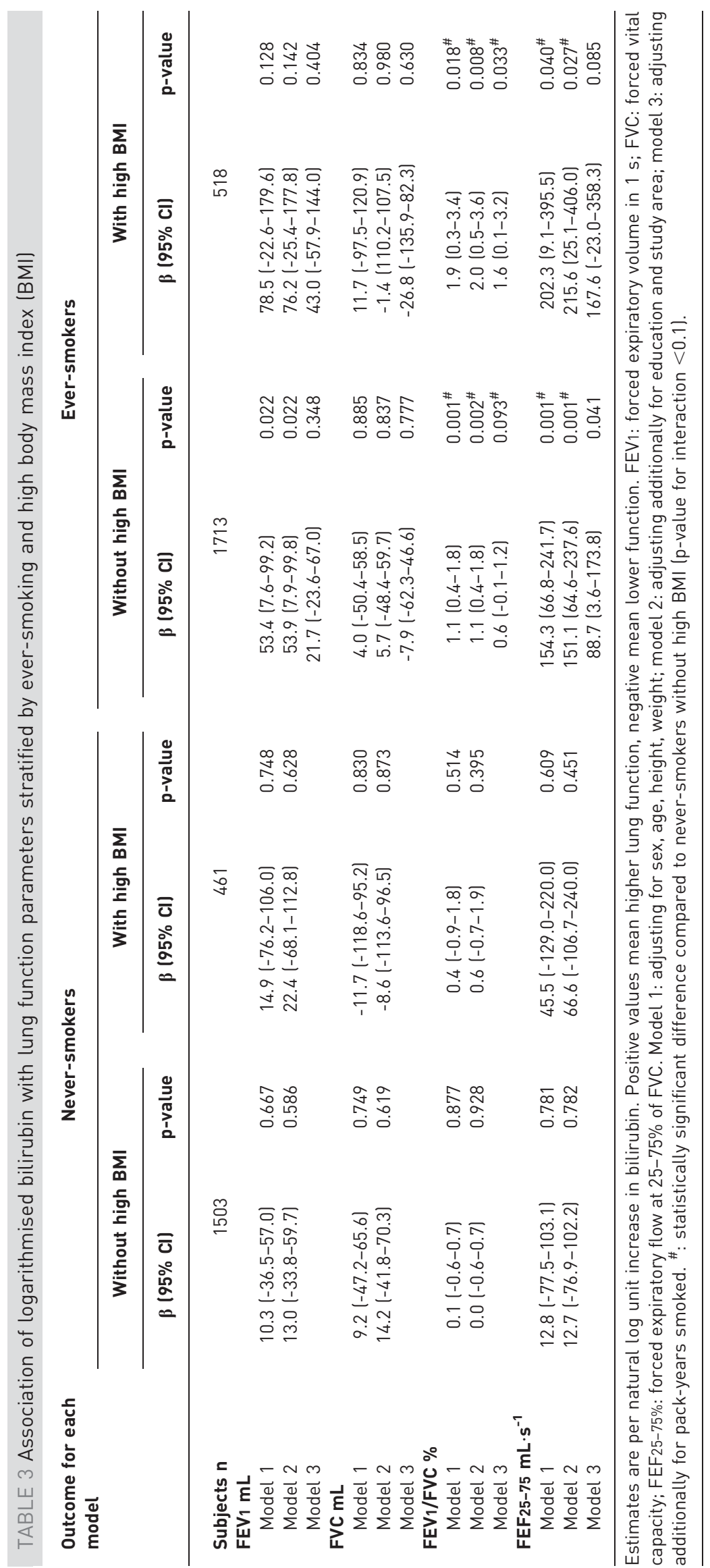


Original analysis

After excluding participants with bilirubin levels above normal

- After excluding participants on asthma medication
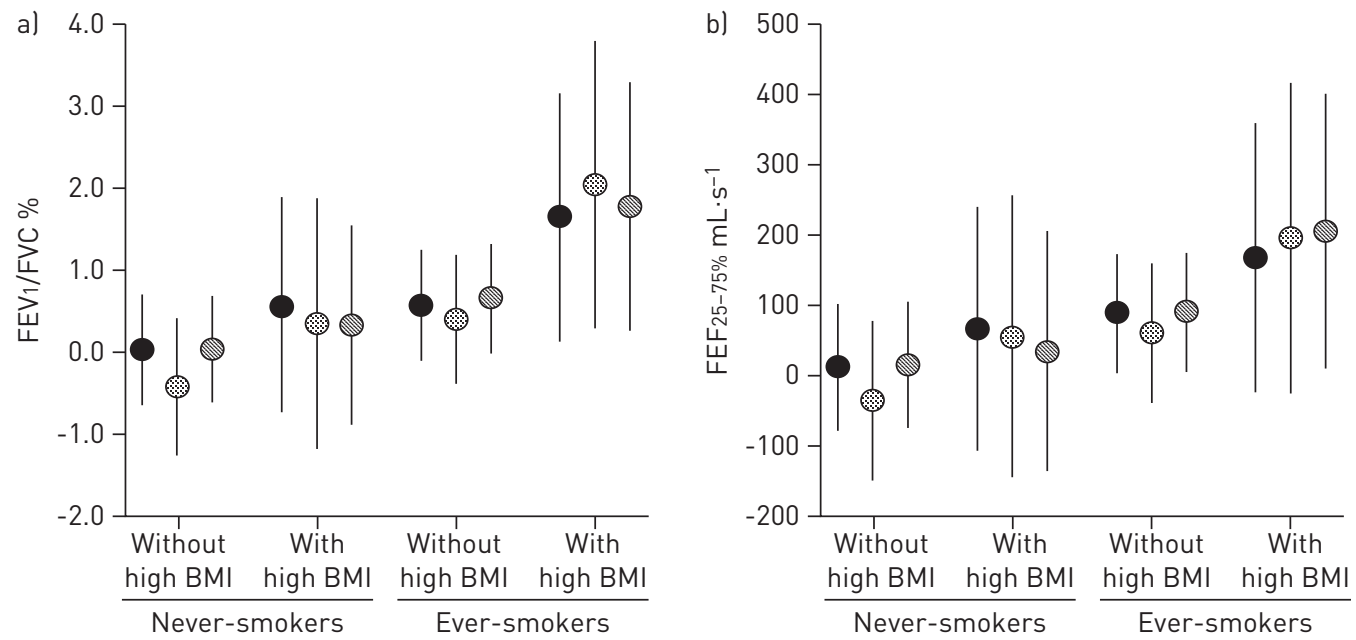

FIGURE 2 Sensitivity analysis of bilirubin associations with a) forced expiratory volume in $1 \mathrm{~s}$ (FEV1)/forced vital capacity (FVC) and b) forced expiratory flow at $25-75 \%$ of FVC (FEF25-75\%). Estimates are indicated per log unit increase in bilirubin. Models adjusted for sex, age, height, weight, education, study area and, in ever-smoker strata, additionally for pack-years smoked. BMI: body mass index.

and $192.5(30.8-354.2) \mathrm{mL} \cdot \mathrm{s}^{-1}$ higher $\mathrm{FEF} 25-75 \%$ than GT/GG carriers after pack-years adjustment. No significant associations were observed for FEV1 and FVC.

\section{Discussion}

In the population-based SAPALDIA study, we found significant positive associations between serum levels of bilirubin and lung function parameters FEV1/FVC and FEF25-75\% after adjusting for the effects of sex, age, education, height and weight, as well as tobacco smoke exposure. When stratifying the study population according to states of increased susceptibility, associations persisted only in participants with

\section{TABLE 4 Associations of rs6742078 genotypes with lung function}

Outcome for each model
Estimates for recessive effects of rs6742078 (allele TT versus GG/GT)

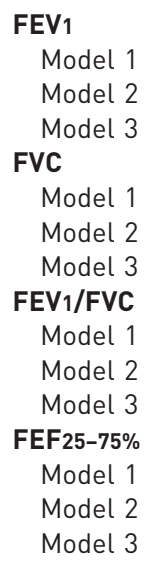

$\begin{array}{ll}27.7(-65.9-121.2) & 0.562 \\ 40.6(-52.6-133.8) & 0.394 \\ 34.7(-57.2-126.7) & 0.459 \\ -58.4(-162.0-45.2) & 0.269 \\ -42.8(-145.8-60.2) & 0.415 \\ -44.3(-147.3-58.7) & 0.399 \\ 1.7(0.4-3.1) & 0.013 \\ 1.8(0.4-3.2) & 0.010 \\ 1.7(0.4-3.0) & 0.013 \\ 190.7(22.9-358.4) & 0.026 \\ 205.6(40.6-370.7) & 0.015 \\ 192.5(30.8-354.2) & 0.020\end{array}$

\#: rs6742078 was coded as recessive effect to correspond with functional effects of the UGT1A1 promoter polymorphism: (TA)7/7 versus (TA)6/6 and (TA)6/7. Genetic data was available for $n=982$ participants. FEV1: forced expiratory volume in $1 \mathrm{~s}$; FVC: forced vital capacity; FEF25-75\%: forced expiratory flow at $25-75 \%$ of FVC; model 1: adjusting for sex, age, height and weight; model 2: additionally adjusting for education and study area; model 3: additionally adjusting for ever-smoking and pack-years smoked. 
high BMI and were increased and significant in ever-smoking strata. Our results were robust to sensitivity analysis, and consistent associations were observed for genetically elevated bilirubin (due to rs6742078).

These findings are coherent with previous epidemiological evidence showing the beneficial effects of elevated serum bilirubin on respiratory outcomes [5-7]. These findings suggest beneficial effects on lung function, but refine their scope to persons with subclinical inflammation and oxidative stress exposure (or cardiopulmonary disease). The significant associations with $\mathrm{FEF} 25-75 \%$ and $\mathrm{FEV} 1 / \mathrm{FVC}$ might point to an inverse relationship with small airway obstruction. Due to their large surface area, small airways are an important compartment of chronic respiratory disease development [26, 27], where subclinical changes often manifest first, and accordingly, strongest associations presented at this site in our general population sample. Smoking adjustment decreased association estimates considerably, which was expected given its known inverse relationship with lung function and serum bilirubin, resulting in positive confounding of the bilirubin lung function association.

We can currently only speculate about the mechanisms by which serum bilirubin might influence lung function. Intracellular mechanisms may be more important in the lung than the known effects in serum and blood vessels, as bilirubin penetrates cell walls at physiological $\mathrm{pH}$ values [28]. Besides scavenging oxidants, bilirubin inhibits membrane-bound nicotinamide adenine dinucleotide phosphate-oxidase, one of the major intracellular sources of reactive oxygen species [29]. Furthermore, bilirubin infusions have been shown to downregulate inflammation in murine lung injury models [30].

Because of the cross-sectional design, the observed associations cannot be interpreted clearly regarding the temporal relationship of outcome and exposure: the findings could arise from a protective effect of preexisting elevated serum bilirubin, preventing tissue damage by inflammatory and oxidative stress reactions, or bilirubin levels could represent a reactive marker of the individual oxidative stress burden and ensuing bilirubin consumption. Our results rather suggest a causal association for different reasons. First, the observed associations between serum bilirubin levels and lung function were consistently replicated in participants with genetically elevated serum bilirubin. Secondly, associations grew stronger across increasing states of subclinical inflammation and oxidative stress (i.e. in persons with high BMI, ever-smokers, or both), and even persisted in persons with cardiopulmonary disease. This would probably not be the case for a reactive biomarker. Finally, we observed similar but less strong associations in the normal range of serum bilirubin.

Our study has few limitations besides its cross-sectional design. Genotyping data was only available in a subset of the population. Our data was potentially affected by measurement error, as lung function was tested only twice, but the resulting misclassification would be random and primarily affect study power. In contrast, our study has several strengths. The population-based design allowed investigation of lung function as a pre-clinical marker of disease processes. The large study sample and detailed data on blood markers, body size and lifestyle factors allowed stratification of the analysis to study associations across subclinical conditions of inflammation and oxidative stress. This offered new insights into the possible health-related effects of bilirubin. Our large study sample also meant that different sensitivity analyses could be conducted. Finally, our study benefited from a standardised spirometry protocol and adherence to strict quality control criteria.

In conclusion, we found significant positive associations of serum bilirubin levels with lung function in persons with increased inflammation and oxidative stress. From a public health perspective, it would be important to clarify whether the observed relationship is causal using prospective studies that include genetic information. Bilirubin could have large preventive and therapeutic potential, given the prevalence of smoking and high BMI in Western populations.

\section{Acknowledgements}

Study directorate was as follows: T. Rochat (pneumology; University Hospitals, Geneva, HUG, Geneva, Switzerland), NM. Probst Hensch (epidemiology/genetic and molecular biology; Swiss Tropical and Public Health Institute Basel, SwissTPH, Geneva, Switzerland), JM. Gaspoz (cardiology; HUG), N. Künzli (epidemiology/exposure; SwissTPH) and C. Schindler (statistics; SwissTPH).

Scientific team was as follows: J.C. Barthélémy (cardiology; Université Jean Monnet, St. Etienne, France), W. Berger (genetic and molecular biology; Universität Zürich, Zurich, Switzerland), R. Bettschart (pneumology; Hirslanden Klinik, Aarau, Switzerland), A. Bircher (allergology; Universitätsspital, Basel, Switzerland), O. Brändli (pneumology; Schweizerische Lungenstiftung, Wald, Switzerland), C. Brombach (nutrition; Zürcher Hochschule für Angewandte Wissenschaften, Zurich), M. Brutsche (pneumology; Kantonsspital St. Gallen, St. Gallen, Switzerland), L. Burdet (pneumology; Hôpital intercantonal de la Broye, Payerne, Switzerland), M. Frey (pneumology; Klinik Barmelweid, Barmelweid, Switzerland), U. Frey (paediatrics; Universitäts-Kinderspital beider Basel, Basel), M.W. Gerbase (pneumology; HUG), D. Gold (epidemiology/cardiology/pneumology; Harvard School of Public Health, Boston, MA, USA), E. de Groot (cardiology; Academic Medical Center, Amsterdam, the Netherlands), W. Karrer (pneumology; Luzerner Höhenklinik Montana, Crans-Montana, Switzerland), R. Keller (pneumology; Klinik Barmelweid, Barmelweid), B. Martin (physical activity; Universität Zürich, Zurich), D. Miedinger (occupational health; Schweizerische 
Unfallversicherungsanstalt SUVA, Luzern), U. Neu (exposure; ProClim, Bern, Switzerland), L. Nicod (pneumology; Centre Hospitalier Universitaire Vaudois, Lausanne, Switzerland), M. Pons (pneumology; Ospedale Regionale di Lugano, Lugano, Switzerland), F. Roche (cardiology; Université Jean Monnet, St. Etienne, France), T. Rothe (pneumology; Zürcher Höhenklinik Davos, Davos Clavadel, Switzerland), E. Russi (pneumology; Universitätsspital Zürich, Zurich), P. Schmid-Grendelmeyer (allergology; Universitätsspital Zürich, Zurich), A. Schmidt-Trucksäss (physical activity; Universität Basel, Basel), A. Turk (pneumology; Zürcher Höhenklinik Wald, Faltigberg-Wald, Switzerland), J. Schwartz (epidemiology; Harvard School of Public Health, Boston), D. Stolz (pneumology; Universitätsspital Basel, Basel), P. Straehl (exposure; Bundesamt für Umwelt BAFU, Bern), J.M. Tschopp (pneumology; Centre Hospitalier du Centre du Valais, Crans-Montana), A. von Eckardstein (clinical chemistry; Universitätsspital Zürich, Zurich) and E. Zemp Stutz (epidemiology; SwissTPH).

The scientific team at coordinating centres was as follows: M. Adam (epidemiology/genetic and molecular biology; SwissTPH), E. Boes (genetic and molecular biology; SwissTPH), PO. Bridevaux (pneumology; HUG), D. Carballo (cardiology; HUG), E. Corradi (epidemiology; SwissTPH), I. Curjuric (epidemiology; SwissTPH), J. Dratva (epidemiology; SwissTPH), A. Di Pasquale (statistics; SwissTPH), E. Dupuis Lozeron (statistics; HUG), M. Germond (statistics; HUG), L. Grize (statistics; SwissTPH), D. Keidel (statistics; SwissTPH), S. Kriemler (physical activity; SwissTPH), A. Kumar (genetic and molecular biology; SwissTPH), M. Imboden (genetic and molecular biology; SwissTPH), N. Maire (statistics; SwissTPH), A. Mehta (epidemiology; SwissTPH), H. Phuleria (exposure; SwissTPH), E. Schaffner (statistics; SwissTPH), GA. Thun (genetic and molecular biology; SwissTPH), A. Ineichen (exposure; SwissTPH), M. Ragettli (epidemiology; SwissTPH), M. Ritter (exposure; SwissTPH), T. Schikowski (epidemiology; SwissTPH), M. Tarantino (statistics; SwissTPH), M. Tsai (epidemiology; SwissTPH) and M. Wanner (physical activity; SwissTPH).

The study could not have been performed without the help of the study participants, technical and administrative support and the medical teams and field workers at the local study sites.

Local fieldworkers were as follows. Study Centre Aarau: S. Brun, G. Giger, M. Sperisen and M. Stahel; Study Centre Basel: C. Bürli, C. Dahler, N. Oertli, I. Harreh, F. Karrer, G. Novicic and N. Wyttenbacher. Study Centre Davos: A. Saner, P. Senn and R. Winzeler. Study Centre Geneva: F. Bonfils, B. Blicharz, C. Landolt and J. Rochat. Study Centre Lugano: S. Boccia, E. Gehrig, M.T. Mandia, G. Solari and B. Viscardi. Study Centre Montana: A.P. Bieri, C. Darioly and M. Maire. Study Centre Payerne: F. Ding, P. Danieli and A. Vonnez. Study Centre Wald: D. Bodmer, E. Hochstrasser, R. Kunz, C. Meier, J. Rakic, U. Schafroth and A. Walder.

Administrative staff were: C. Gabriel (HUG) and R. Gutknecht (SwissTPH).

\section{References}

1 Kronenberg F. Association of bilirubin with cardiovascular outcomes: more hype than substance? Circ Cardiovasc Genet 2010; 3: 308-310.

2 Schwertner HA, Vitek L. Gilbert syndrome, UGT1A1²8 allele, and cardiovascular disease risk: possible protective effects and therapeutic applications of bilirubin. Atherosclerosis 2008; 198: 1-11.

3 Lin JP, Vitek L, Schwertner HA. Serum bilirubin and genes controlling bilirubin concentrations as biomarkers for cardiovascular disease. Clin Chem 2010; 56: 1535-1543.

4 Stocker R, Yamamoto Y, McDonagh AF, et al. Bilirubin is an antioxidant of possible physiological importance. Science 1987; 235: 1043-1046.

5 Horsfall LJ, Rait G, Walters K, et al. Serum bilirubin and risk of respiratory disease and death. JAMA 2011; 305: 691-697.

6 Temme EH, Zhang J, Schouten EG, et al. Serum bilirubin and 10-year mortality risk in a Belgian population. Cancer Causes Control 2001; 12: 887-894.

7 Breimer LH, Wannamethee G, Ebrahim S, et al. Serum bilirubin and risk of ischemic heart disease in middle-aged British men. Clin Chem 1995; 41: 1504-1508.

8 Rodrigues C, Costa E, Vieira E, et al. Bilirubin dependence on UGT1A1 polymorphisms, hemoglobin, fasting time and body mass index. Am J Med Sci 2012; 343: 114-118.

9 Jo J, Kimm H, Yun JE, et al. Cigarette smoking and serum bilirubin subtypes in healthy Korean men: the Korea Medical Institute study. J Prev Med Public Health 2012; 45: 105-112.

10 Johnson AD, Kavousi M, Smith AV, et al. Genome-wide association meta-analysis for total serum bilirubin levels. Hum Mol Genet 2009; 18: 2700-2710.

11 Bosma PJ, Chowdhury JR, Bakker C, et al. The genetic basis of the reduced expression of bilirubin UDPglucuronosyltransferase 1 in Gilbert's syndrome. N Engl J Med 1995; 333: 1171-1175.

12 Lin JP, O’Donnell CJ, Schwaiger JP, et al. Association between the UGT1A1*28 allele, bilirubin levels, and coronary heart disease in the Framingham Heart Study. Circulation 2006; 114: 1476-1481.

13 Lin R, Wang Y, Fu W, et al. Common variants of four bilirubin metabolism genes and their association with serum bilirubin and coronary artery disease in Chinese Han population. Pharmacogenet Genomics 2009; 19: 310-318.

14 Bosma PJ, van der Meer IM, Bakker CT, et al. UGT1A1 28 allele and coronary heart disease: the Rotterdam Study. Clin Chem 2003; 49: 1180-1181.

15 Ekblom K, Marklund SL, Jansson JH, et al. Plasma bilirubin and UGT1A1 ${ }^{\star} 28$ are not protective factors against first-time myocardial infarction in a prospective, nested case-referent setting. Circ Cardiovasc Genet 2010; 3: 340-347.

16 Gajdos V, Petit FM, Perret C, et al. Further evidence that the UGT1A1 ${ }^{2} 28$ allele is not associated with coronary heart disease: The ECTIM Study. Clin Chem 2006; 52: 2313-2314.

17 Lingenhel A, Kollerits B, Schwaiger JP, et al. Serum bilirubin levels, UGT1A1 polymorphisms and risk for coronary artery disease. Exp Gerontol 2008; 43: 1102-1107.

18 Rantner B, Kollerits B, Anderwald-Stadler M, et al. Association between the UGT1A1 TA-repeat polymorphism and bilirubin concentration in patients with intermittent claudication: results from the CAVASIC study. Clin Chem 2008; 54: 851-857. 
19 Stender S, Frikke-Schmidt R, Nordestgaard BG, et al. Genetically elevated bilirubin and risk of ischaemic heart disease: three Mendelian randomization studies and a meta-analysis. J Intern Med 2013; 273: 59-68.

20 Ackermann-Liebrich U, Kuna-Dibbert B, Probst-Hensch NM, et al. Follow-up of the Swiss Cohort Study on Air Pollution and Lung Diseases in Adults (SAPALDIA 2) 1991-2003: methods and characterization of participants. Soz Praventivmed 2005; 50: 245-263.

21 American Thoracic Society. Standardization of spirometry-1987 update. Statement of the American Thoracic Society. Am Rev Respir Dis 1987; 136: 1285-1298.

22 Wahlefeld AWHG, Bernt E. Modification of the Malloy-Evelyn method for a simple, reliable determination of total bilirubin in serum. Scand J Clin Lab Invest 1972; 29: Suppl. 126, abstract 11.2.

23 Moffatt MF, Gut IG, Demenais F, et al. A large-scale, consortium-based genomewide association study of asthma. New Engl J Med 2010; 363: 1211-1221.

24 Imboden M, Bouzigon E, Curjuric I, et al. Genome-wide association study of lung function decline in adults with and without asthma. J Allergy Clin Immunol 2012; 129: 1218-1228.

25 Bochud M, Rousson V. Usefulness of Mendelian randomization in observational epidemiology. Int J Environ Res Public Health 2010; 7: 711-728.

26 Hamid Q. Pathogenesis of small airways in asthma. Respiration 2012; 84: 4-11.

27 Burgel PR, Bourdin A, Chanez P, et al. Update on the roles of distal airways in COPD. Eur Respir Rev 2011; 20: $7-22$.

28 Zucker SD, Goessling W, Hoppin AG. Unconjugated bilirubin exhibits spontaneous diffusion through model lipid bilayers and native hepatocyte membranes. J Biol Chem 1999; 274: 10852-10862.

29 McCarty MF. "Iatrogenic Gilbert syndrome" - a strategy for reducing vascular and cancer risk by increasing plasma unconjugated bilirubin. Med Hypotheses 2007; 69: 974-994.

30 Ryter SW. Bile pigments in pulmonary and vascular disease. Front Pharmacol 2012; 3: 39. 